\section{Feline lymphoma presenting with pleural effusion - response to treatment and outcome in 13 cats: a retrospective case series}

\section{Aaron Harper, Sarah Mason}

University of Liverpool, Liverpool, UK

Pleural effusion may be associated with feline lymphoma secondary to both intrathoracic and multicentric disease. Affected cats may not be treated if effusion is perceived as a negative prognostic indicator by clinicians. The aim of this study is to report outcomes of cats with lymphoma with pleural effusion on presentation that received chemotherapy; and to identify factors associated with survival.

Case records were retrospectively reviewed. Inclusion criteria were: presence of pleural effusion at presentation, cytological/histological diagnosis of lymphoma, staging (radiographs, ultrasound, haematology + biochemistry, FIV/FeLV), and treatment with chemotherapy. Prognostic factors analysed were age, duration of clinical signs, haematocrit, respiratory vs nonrespiratory presenting signs, rescue chemotherapy, and whether thoracocentesis was performed.

13 cats were included. Median age was 8 years (range 0.5-13.9). Clinical signs included dyspnoea/tachypnoea (8/13) weight loss (4/13) and anorexia (4/13). Four cats presented for non-respiratory signs, and pleural effusion was an incidental finding. Median duration of clinical signs prior to presentation was 14 days (range $1-30$ ). 2/13 cats tested FeLV positive. No cats were FIV positive. 7/13 cats had therapeutic thoracocentesis performed, and the median number of drainages was 2 . All cats received high-dose COP chemotherapy. Clinical response was observed in 9/13 cats. When relapse occurred, or for nonresponders, other chemotherapy agents including lomustine, cytarabine and epirubicin were used. Median survival (MST) for all cats was 66 days (range 4-934) with 2 cats alive at the time of writing (967 and 203 days). MST for responding and nonresponding cats was 70 and 12 days respectively. Response to treatment was significantly associated with MST $(p=<0.0001)$. Cats presenting with respiratory signs $(p=0.0313)$ and receiving thoracocentesis $(0.0285)$ had significantly improved MST (70 days vs 17.5 days and 87.5 days vs 20 days respectively) compared with those that did not. 3/4 cats which presented with non-respiratory signs did not respond to chemotherapy. Duration of clinical signs, sex, rescue treatment and haematocrit were not significantly associated with survival. Cats less than the median age of presentation (8 years) trended towards significance for increased survival $(p=0.089)$

Overall, this small cohort of cats with lymphoma-associated pleural effusion had poor MST compared with previously reports for mediastinal lymphoma (373 days), although individual cats achieved long survivals with treatment. Further studies in larger cohorts are required to assess the significance of pleural effusion as a prognostic factor in feline lymphoma.

\section{A study investigating the epidemiological factors influencing the development of osteosarcoma in UK Rottweilers}

\section{Shareen Akhtar ${ }^{1}$, David Gardner ${ }^{1}$, Tim Scase $^{4}$, Trevor Whitbread ${ }^{3}$, Annalize Ide ${ }^{2}$, Mark Dunning ${ }^{1}$}

\author{
School of Veterinary Medicine and Science, University of Nottingham, \\ Leicestershire, UK \\ Finn Pathologists, Norfolk, UK \\ Abbey Vet Services, Devon, UK \\ Bridge Pathology, Bristol, UK
}

Osteosarcoma is the most common primary bone tumour in dogs. Despite advances in veterinary oncology our ability to effectively treat osteosarcoma remains limited. Most dogs die from metastatic disease within 1-2 years of diagnosis. Rottweilers are reported to have a higher incidence compared with other breeds, although the reasons for this have not been investigated in the UK cohort.

Submission data from UK histopathology laboratories were reviewed. This demonstrated that Rottweilers were amongst the most frequent of all submissions of osteosarcoma samples (11\%). The proportion of osteosarcoma submissions in Rottweilers relative to the overall submissions to the laboratories was higher than for other breeds (1.5\% vs. <0.5\%). This suggested an increased incidence of osteosarcoma in UK
Rottweilers. As a result of this a questionnaire was designed to identify possible risk factors for the development of osteosarcoma. This was based on the lifestyle and management practices and was distributed at dog shows, via breed groups and social media fora. A total of 749 questionnaires were completed, 100 of which were from dogs affected by osteosarcoma. Factors assessed included age, sex, neuter status, body condition, exercise levels, history of orthopaedic disease, taking of prescription medications and vaccination history. A regression model was developed to determine the factors influencing the development of osteosarcoma. The results indicated that neutering increased the risk of developing osteosarcoma $(\mathrm{P}<0.01)$. Although a trend was noted for a higher risk in males, this was not a significant association. The risk of developing osteosarcoma was greater in Rottweilers that were neutered earlier $(\mathrm{P}<0.01)$. An overall lifetime risk of $24-30 \%$ was noted for animals of either sex being neutered before 6 months of age. As the age at neutering increased the relative risk fell with an $11 \%$ risk if neutered at 84 months. A history of orthopaedic disease was also shown to significantly influence the development of osteosarcoma $(\mathrm{P}<0.01)$. The remaining risk factors investigated were found not to be significant - these included vaccination status, obesity and exercise duration, frequency and intensity.

Our data confirm that UK Rottweilers are at increased risk of developing osteosarcoma relative to other UK breeds. It appears that early neutering strongly influences the development of osteosarcoma, as does a history of orthopaedic disease. It would seem reasonable to use this information to guide the early management of the UK Rottweiler cohort. 